\title{
Features of methods for monitoring the fuel cladding tightness in lead-cooled fast breeder reactors ${ }^{*}$
}

\author{
Anastasiya V. Dragunova ${ }^{1,2}$, Mikhail S. Morkin ${ }^{1}$, Vladimir V. Perevezentsev² \\ 1 NIKIET JCS, 2/8 Malaya Krasnoselskaya Str., 107140 Moscow, Russia \\ 2 Bauman Moscow State Technical University, 5, bldg. 1 Vtoraya Baumanskaya Str., 105005 Moscow, Russia \\ Corresponding author: Anastasiya V. Dragunova (a-dragunova@bk.ru)
}

Academic editor: Yury Kazansky • Received 20 March 2021 Accepted 12 September 2021 • Published 17 December 2021

Citation: Dragunova AV, Morkin MS, Perevezentsev VV (2021) Features of methods for monitoring the fuel cladding tightness in lead-cooled fast breeder reactors. Nuclear Energy and Technology 7(4): 319-325. https://doi.org/10.3897/nucet.7.78372

\begin{abstract}
To timely detect failed fuel elements, a reactor plant should be equipped with a fuel cladding tightness monitoring system (FCTMS). In reactors using a heavy liquid-metal coolant (HLMC), the most efficient way to monitor the fuel cladding tightness is by detecting gaseous fission products (GFP).

The article describes the basic principles of constructing a FCTMS in liquid-metal-cooled reactors based on the detection of fission products and delayed neutrons. It is noted that in a reactor plant using a HLMC the fuel cladding tightness is the most efficiently monitored by detecting GFPs. The authors analyze various aspects of the behavior of fission products in a liquid-metal-cooled reactor, such as the movement of GFPs in dissolved and bubble form along the circuit, the sorption of volatile FPs in the lead coolant (LC) and on the surfaces of structural elements, degassing of the GFPs dissolved in the LC, and filtration of cover gas from aerosol particles of different nature.

In addition, a general description is given of the conditions for the transfer of GFPs in a LC environment of the reactor being developed. Finally, a mathematical model is presented that makes it possible to determine the calculated activity of reference radionuclides in each reactor unit at any time after the fuel element tightness failure. Based on this model, methods for monitoring the fuel cladding tightness by the gas activity in the gas volumes of the reactor plant will be proposed.
\end{abstract}

\section{Keywords}

BREST, fuel cladding tightness monitoring, lead coolant, fuel element, failed cladding, fission products, inert radioactive gases, two-phase flows, bubbling up, diffusion, degassing, mathematical model

\section{Introduction}

The lead-cooled BREST-OD-300 reactor plant using nitride uranium-plutonium fuel is being developed in order to substantiate the possibility of deploying large-scale nuclear power industry based on fast reactors, which could solve the problems of removing restrictions on fuel resources, eliminating severe accidents at nuclear power plants and closing the nuclear fuel cycle to ensure economic competitiveness (Adamov et al. 2020).

The main goal of ensuring safety for modern reactor plants is to prevent the release of radioactive products

* Russian text published: Izvestiya vuzov. Yadernaya Energetika (ISSN 0204-3327), 2021, n. 3, pp. 84-96. 
beyond the physical barriers, which include the fuel matrix, fuel element cladding, reactor coolant boundary, etc. (Adamov et al. 2005).

Due to the limitation of the dose load on the population, the project regulates the maximum possible number (in \%) of gas-leaking and having fuel-to-coolant contact fuel elements, since when the fuel element tightness fails, a certain amount of fission products goes into the coolant, increasing its radioactivity.

In accordance with modern standards in the field of nuclear power, in order to determine the number of failed fuel elements, their location and the type of cladding failure, the BREST reactor facility should be provided with a fuel cladding tightness monitoring system (FCTMS), developed on the basis of the experience in designing and operating such systems for existing types of fast reactors (NP-082-07 2008).

\section{Fuel cladding tightness monitoring in liquid-metal-cooled reactors}

In BN-type fast reactors, the fuel cladding tightness monitoring is based on the detection of fission products and delayed neutrons in the coolant and the cover gas of the primary circuit (Lukyanov and Albutova 2017). The sector system, designed for continuous measurement of the delayed neutron flux density in order to register failures of the "direct fuel-to-coolant contact" type, makes it possible to determine the sector of the location of failed fuel assemblies within 1/6 of the core. The readings of the gas system, which serves for continuous measurement of the activity of gaseous fission products in the reactor blanketing gas, are used to register failures of the "gas leak" type and areas of the core with failed fuel assemblies. To isolate reference radionuclides in the coolant, spectrometric methods with freezing and preliminary holding of a sodium sample and the method of sorption of cesium isotopes in an adsorber based on reactor graphite are used (Lukyanov et al. 2018).

The operational principle of the FCTMS of a nuclear power plant (NPP) using a lead-bismuth coolant (LBC) is to take a part of the cover gas into the measuring loop, clean it from aerosols to reduce background aerosol activity and perform a gamma-spectrometric analysis of the isotopic composition of radioactive noble gases (RNG) in the measuring tank (Dedul et al. 2009).

The dominant contribution to the activity of the coolant and the cover gas is made by activation products and RNGs, respectively; the latter is explained by the high distribution coefficient of these gases between the gaseous medium and the liquid metal. The fuel cladding tightness failure leads to an increase in the content of fission products both in the coolant and in the gas circuit.

For the SVBR-100 (lead-bismuth fast reactor) with an intermediate neutron spectrum, it was shown in (Gonchar 2018) that at a surface contamination density of fuel elements equal to $1 \times 10^{-8} \mathrm{~g} / \mathrm{cm}^{2}$, the total volumetric activity of the RNGs is close to the upper limit of the normal state of the core, when all the fuel elements are leak-tight. In this case, the greatest contribution to the background activity is made by the surface contamination of the fuel element claddings with the fuel composition. At the minimum density of the surface contamination of the fuel element claddings, the main contribution to the background activity is made by fast neutron fission of ${ }^{238} \mathrm{U}$ contained in the lead-bismuth coolant.

The background source of delayed neutrons in the coolant, which determines the threshold sensitivity for assessing the size of the failure of the "direct fuel-to-coolant contact" type, with the above weight fraction of uranium in the lead-bismuth coolant corresponds to about $10-15 \mathrm{~cm}^{2}$ of the total open surface area of the fuel in the core (Gonchar 2018).

Thus, in special literature about liquid-metal-cooled reactors, gamma spectrometry of the cover gas and measurement of the delayed neutron flux in the coolant are considered as the fuel cladding tightness monitoring methods. To justify the specific technical solutions of the FCTMS for lead-cooled reactors, it is necessary to take into account the peculiarities of the transfer of radionuclides in the lead coolant and the cover gas. Let us consider the basic relationships used in the design justification of such a system.

\section{Features of fuel cladding tightness monitoring in lead-cooled reactors}

As the fuel burns up, the pressure under the fuel element cladding increases due to the accumulation of both stable and radioactive isotopes of the GFPs formed during the fission of fuel nuclei and the radioactive decay of fission fragments.

When a failure occurs in a fuel element before a certain degree of fuel burnup due to the high density of lead, its pressure exceeds the gas pressure inside the fuel element, which leads to the lead leakage into the fuel element under the condition (Putilov 1963)

$$
d_{\text {fail }}{ }^{\text {crack }}>2 \sigma_{\mathrm{Pb}} / \Delta p,
$$

where $d_{\text {fail }}^{\text {crack }}$ is the characteristic size of the failure (crack); $\sigma_{\mathrm{Pb}}$ is the surface tension coefficient of lead, $\mathrm{N} / \mathrm{m}$; $\Delta p$ is the pressure drop across the rupture.

When the gas pressure under the fuel element cladding reaches its critical value, single bubbles of the GFPs will emerge into the coolant volume. If a failure occurs at a time when the gas pressure in the fuel element is higher than the coolant pressure, the GFPs that form the excess pressure leave the fuel element in a jet within a short time, after which they will emerge as single bubbles as they are produced.

Due to the low speed of the coolant and its large volume in the reactor, it is possible to track only large failures using delayed neutrons; therefore, the most effective way to monitor the fuel cladding tightness in a lead-cooled reactor is by detecting the GFPs. 


\section{Behavior of fission products in the coolant}

\section{Dissolved GFPs}

When substantiating the efficiency of detecting leaks of fuel elements by measuring the activity of the GFPs, it is necessary to consider the physical processes that determine their transfer in the coolant as well as other reactor media and auxiliary systems. Gas can flow from a leaky fuel element into the coolant both in the dissolved form and as bubbles of various sizes. To assess the possible content of dissolved GFPs in the lead coolant, it is required to have data on the solubility (saturation concentration) of gases in it at operating temperatures in the circuit.

In (Skovorodko and Mozgovoy 2010), the theoretical values of the solubility of xenon and krypton in lead were estimated at temperatures up to $1500 \mathrm{~K}$. For the solubility of krypton or xenon in lead at a gas pressure of $0.1 \mathrm{MPa}$, the following dependence was proposed, which was recommended in (Solubility data series 1979):

$$
\ln (S)=A+B / \tau+C \times \ln (\tau)+D \times \tau
$$

where $S$ is the solubility in moles of gas per mole of lead; $\tau=T / 1000, T$ is the temperature, $\mathrm{K} ; A, B, C, D$ are the coefficients presented in Table 1 (Skovorodko and Mozgovoy 2010).

Table 1. Coefficients for calculating the solubility of krypton and xenon at a pressure of $0.1 \mathrm{MPa}$

\begin{tabular}{ccccc}
\hline & $\boldsymbol{A}$ & $\boldsymbol{B}$ & $\boldsymbol{C}$ & $\boldsymbol{D}$ \\
\hline $\mathrm{Kr}$ & -17.41852 & 2.17779 & 9.06075 & -0.81210 \\
$\mathrm{Xe}$ & -20.77263 & 3.54320 & 11.70382 & -1.06850 \\
\hline
\end{tabular}

According to the "hole" model, the solubility of inert gases in liquid metals linearly depends on pressure (Skovorodko and Mozgovoy 2010):

$$
S_{p}=\left(p / p_{0}\right) \times S_{10}
$$

where $S_{10}, S_{p}$ is the solubility of inert gases in a HLMC at a calculated pressure $p_{0}=0,1 \mathrm{MPa}$ and an equilibrium pressure in the system $p$ in $\mathrm{MPa}$, respectively.

For the temperature and pressure of lead at the outlet from the core of the BREST-OD-300 reactor, the solubility of krypton and xenon is $182 \times 10^{-9}$ and $16 \times 10^{-9} \mathrm{~mol}$ of gas/mol of $\mathrm{Pb}$, respectively.

\section{Bubbles}

In addition to the dissolved form, the GFPs are present in the coolant as bubbles of various sizes. Evaluation of the maximum possible diameter of a gas bubble in the lead coolant flow according to the formula, verified by the results of testing the model of the gas lift probe on the facility at NIKIET JSC (Vereschagina et al. 2020), gives the maximum bubble diameter in the lead coolant flow at operating parameters in the BREST-OD-300 reactor plant equal to $18 \mathrm{~mm}$ :

$$
d_{\max }=2 L^{2 / 3}\left(2 \sigma / \rho_{1}\right)^{3 / 5} U_{1}^{-6 / 5}\left(\rho_{1} / \rho_{g}\right)^{1 / 5},
$$

where $L$ is the characteristic size of the channel; $\sigma$ is the surface tension coefficient ; $\rho_{1}$ is the LC density; $\rho_{g}$ is the gas density; $U_{1}$ is the $\mathrm{LC}$ flow rate.

In (Fedotovskiy et al. 2007), a mathematical model of bubble coagulation at the blowdown of a gas-liquid flow from a jet injector into a channel with a ST flow is presented, which makes it possible to calculate the bubble size distribution at any time:

$$
C_{i}(t)=\frac{C_{i 0}}{\left(1+0,5 k C_{0} t\right)^{2}}+\sum_{k=1}^{i-1} \sum_{j=1}^{i-1} C_{j 0} C_{i-j, k-1} \frac{0,5(k \cdot t)^{k-1}}{\left(1+0,5 k C_{0} t\right)^{k+1}}(5)
$$

where $C_{i 0}$ is the initial concentration of bubbles of the $i$-th size; $k$ is the constant of coagulation, which depends on its mechanism.

In turbulent flows, there are three coagulation mechanisms: gradient (with a large gradient of the jet velocity), turbulent and inertial.

The fastest coalescence of bubbles occurs at the initial section of the jet. Further, the polydisperse spectrum of bubble sizes degenerates into a monodisperse spectrum corresponding to the maximum possible bubble size in the flow.

The dependence of the bubble rise velocity on its diameter is determined by the Peebles and Garber correlations (Wallis G 1972), which correspond to the case of the negligible gas density compared to the liquid density. The range of applicability of each equation is determined by the dimensionless complex presented in Table 2:

$$
G_{1}=g \mu_{f}^{4} /\left(\rho_{f} \sigma^{3}\right)
$$

where $g$ is the acceleration of gravity; $\mu_{f}$ is the dynamic viscosity of the liquid; $\rho_{f}$ is the density of the liquid; $\sigma$ is the surface tension coefficient.

The dependence of the bubble rise velocity in the LC on its size, calculated by the ratios from Table 2 , is shown in Fig. 1.

Table 2. Terminal rise velocity of single gas bubbles in liquid

\begin{tabular}{lcc}
\hline \multicolumn{1}{c}{ Area } & Terminal velocity & Range of applicability \\
\hline 1 & $w=0.33 g^{0,76}\left(\rho_{f} / \mu_{f}\right)^{0.52}(d / 2)^{1.28}$ & $2<\operatorname{Re}<4.02 G_{1}^{-0.0214}$ \\
2 & $w=1.35\left[2 \sigma /\left(\rho_{f} d\right)\right]^{0.5}$ & $4,02 G_{1}^{-0,0214}<\operatorname{Re}<3.1 G_{1}^{-0.25}$ \\
3 & $w=1.18\left[g \sigma / \rho_{f}\right]^{0.25}$ & $\operatorname{Re}>3.1 G_{1}^{-0.25}$ \\
\hline
\end{tabular}

\section{Sorption of volatile fission products}

A crack in the fuel element cladding releases volatile FPs, primarily isotopes of iodine and cesium, which can be contained in bubbles in the form of vapors or aerosols; in the case of large defects, they can be washed off from the fuel 


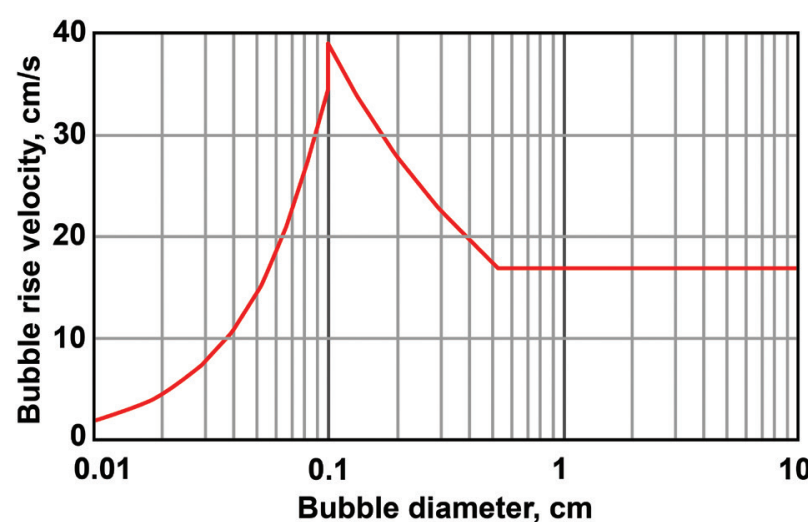

Figure 1. Dependence of the bubble rise velocity on its size.

surface and the inner side of the cladding, and also enter the coolant by direct recoil. Once in the circulation circuit, depending on the nature of the coolant and the oxygen content in it, the FPs are distributed between the solid surfaces of the circuit and the coolant (Naumov and Konovalov 2018).

To monitor the fuel cladding tightness in lead-cooled reactors, it is recommended that radionuclides that are readily soluble in the coolant and determined in the sample, for example, ${ }^{106} \mathrm{Ru}$ and ${ }^{125} \mathrm{Sb}$, should be considered as reference ones. On the other hand, there are proposals on the applicability of cesium isotopes as reference radionuclides, which are insoluble in the melt and well sorbed on the surfaces of structural elements.

The cesium transfer factors found during experimental studies in the form of oxide deposits on the container surface at a mass oxygen content in the $\mathrm{Pb}-\mathrm{Bi}$ alloy of $5 \times 10^{-40} \%$ and an alloy temperature of $500^{\circ} \mathrm{C}$ are presented in Table 3 (Naumov and Konovalov 2018).

Table 3. Data on the distribution of cesium oxide deposits between the walls of the container and the $\mathrm{Pb}-\mathrm{Bi}$ melt

\begin{tabular}{lccc}
\hline \multirow{2}{*}{ Source } & $\begin{array}{c}\text { Holding } \\
\text { time, }\end{array}$ & \multicolumn{2}{c}{ Share of activity, \% } \\
\cline { 3 - 5 } & days & Melt & Oxide deposits \\
\hline Radionuclides released into the melt from & 30 & 4 & 96 \\
$\mathrm{UO}_{2}$ pellets irradiated in a fast reactor & 47 & 4 & 96 \\
& 47 & 6 & 94 \\
\hline Radionuclides released into the melt from & 30 & 73 & 27 \\
$\mathrm{UBe}_{13}$ pellets irradiated in a fast reactor & & & \\
\hline
\end{tabular}

According to (SanPiN 2.6.1.24-03), the radiation monitoring system should provide control of reference radionuclides characterizing the tightness of protective barriers, including ${ }^{134} \mathrm{Cs}$ and ${ }^{137} \mathrm{Cs}$. In this regard, the reactor plant should be equipped with a system that makes it possible to determine the amount of cesium in the coolant sample.

\section{Degassing gaseous fission products in a lead coolant}

After entering the coolant surface during its mixing, the dissolved GFPs leave the coolant into the gas system through the interfacial area due to diffusion processes in the boundary layer. The rate of their release is determined by the relative rate of degassing, i.e., the ratio of the rate of release of the $i$-th isotope to its activity in the coolant.

In (Mikhailov and Gonchar 2015), it is recommended to calculate the diffusion coefficient of RNGs in a coolant using the Einstein-Stokes formula (Babichev et al. 1991), since the results of calculations in this case agree with experimental data:

$$
D=D=k T /(6 \pi r \mu)
$$

where $k=1.38 \times 10^{-23} \mathrm{~J} / \mathrm{K}$ is the Boltzmann constant; $T$ is the coolant temperature, $\mathrm{K} ; r$ is the gas atom radius, $\mathrm{m} ; \mu$ is the LMC dynamic density, $\mathrm{Pa} \times \mathrm{s}$.

According to (Mikhailov and Gonchar 2015), an approximate estimate of the relative degassing rate can be performed as follows:

$$
\begin{aligned}
v_{0} & =D \times S_{\mathrm{m}} /\left(\delta_{\mathrm{bl}} \times V_{\text {cool }}\right)=k T S_{\mathrm{m}} /\left(6 \pi r \mu \delta_{\mathrm{bl}} V_{\text {cool }}\right)= \\
& =k T S_{\mathrm{m}} /\left[6(\pi \mu)^{3 / 2} r V_{\text {cool }}\right] \times\left[\rho^{5} g^{3} /(64 \sigma)\right]^{0,125},
\end{aligned}
$$

where $D$ is the diffusion coefficient of gas in the coolant, $\mathrm{cm}^{2} / \mathrm{s} ; S_{\mathrm{m}}$ is the area of the coolant mirror in the buffer tank, $\mathrm{m}^{2} ; \delta_{\mathrm{bl}}$ is the boundary layer thickness, $\mathrm{m} ; V_{\text {cool }}$ is the coolant volume, $\mathrm{m}^{3} ; \rho$ is the coolant density, $\mathrm{kg} / \mathrm{m}^{3} ; \sigma$ is the surface tension coefficient, $\mathrm{N} / \mathrm{m}$.

The diffusion coefficient of GFPs in the LC can be approximately estimated using the data given in the reference book (Handbook on Lead-Bismuth Eutectic Alloy), which indicates various temperature dependences for the oxygen diffusion coefficient. The widest possible range of temperatures of applicability (673-1273 K) for the Gromov formula:

$$
D\left[\mathrm{~cm}^{2} / \mathrm{s}\right]=6.6 \times 10^{-5} \times \exp [-16158 /(R \times T)],
$$

where $R=8,314 \mathrm{~J} /(\mathrm{mol} \times \mathrm{K})$ is the universal gas constant.

To experimentally confirm the rates of release from lead into the gaseous environment for specific radionuclides under specific conditions, a complex of lead-cooled loop installations was designed and created at JSC IRM, on which the processes of migration of fuel fission products and activation products of a liquid metal coolant were studied (Vasyukhno et al. 2017).

\section{Behavior of aerosols in the cover gas}

Physicochemical processes occurring in the circuits of a nuclear power plant with a circulating HLMC lead to the formation of fine particles of various nature with their subsequent release into gas volumes in the form of aerosols (Martynov et al. 2007). To study the characteristics of aerosols, experimental installations were created that simulate real conditions and modes of operation with HLMCs. It was noted that the aerosols formed during the 
evaporation of lead are characterized by high polydispersion. Generalized data on the concentration of particles at different distances from the lead surface are presented in Table 4.

Table 4. Generalized data on the concentration of particles depending on the height of sampling of the analyzed gas during evaporation

\begin{tabular}{lcccc}
\hline \multirow{2}{*}{$\begin{array}{c}\text { Melt } \\
\text { temperature, } \\
{ }^{\circ} \mathbf{C}\end{array}$} & $\begin{array}{c}\text { Characteristic } \\
\text { particle size, } \\
\boldsymbol{\mu m}\end{array}$ & \multicolumn{3}{c}{ Total mass concentration, $\mathbf{~ m g} / \mathbf{m}^{3}$} \\
\cline { 3 - 5 } & & $\begin{array}{c}\text { Sampling point height above the lead } \\
\text { surface, } \mathbf{~ m m}\end{array}$ \\
\hline 450 & 0.1 & 0.03 & 0.02 & $>2$ \\
500 & 0.1 & 0.05 & 0.03 & $>2$ \\
600 & 0.4 & 0.13 & 0.12 & $0.4-0.5$ \\
\hline
\end{tabular}

If no special measures are taken to clean the circulating cover gas from aerosol particles, adverse consequences are possible associated with an increase in the hydraulic resistance of the channels, a decrease in the performance of control and measuring equipment, as well as a deterioration in the radiation situation due to the deposition of impurities on the elements of gas systems, i.e., sources of ionizing radiation. In (Posazhennikov et al. 2015), it is proposed to use filtering devices with several purification sections: a high-temperature one - to purify gas at the reactor outlet from large aerosol particles larger than 5 microns at a temperature of $450-600^{\circ} \mathrm{C}$ and a low-temperature one - to perform final gas purification on the cold side of the circuit $\left(20-40^{\circ} \mathrm{C}\right)$. As a high-temperature filtering material, a needled metal fabric was considered, which was obtained by the method of extraction (i.e., extraction using a solvent) of a hanging melt droplet. Its efficiency turned out to be close to $35 \%$ for particles with a size of $0.2-1.0 \mu \mathrm{m}$ and up to $86 \%$ for larger particles. Tests of glass papers produced by JSC VNII TsBP and Hollingsworth and Vose Company for the efficiency of gas cleaning from particles larger than $0.3 \mu \mathrm{m}$ showed high results, i.e., $99.95 \%$ and $99.96 \%$, respectively.

\section{Fuel cladding tightness monitoring for the BREST reactor}

Taking into account all of the above, the developed project of a promising lead-cooled reactor provides for means of continuous gas extraction from the volume of the gas cavities of the reactor section to control its total activity and nuclide composition. The radionuclides dissolved in the LC are transferred in the circuit with the coolant flow and exit into the cover gas from free surfaces above the core, near the steam generators (SG) and main circulation pumps (MCP). The expected effect of this process on the measured gas activity can be calculated using a mathematical model that takes into account the transfer of nuclides, in which the GFPs released from the leaking fuel elements enter the LC passing through the core, then into the other parts of the lead circulation loop and into the gas volumes of the reactor section. The model reproduces the lead circulation circuit as a sequence of transitions from one selected volume to another, which is shown in Fig. 2. This approach is described in the monograph (Veselkin and Yegorov 1976).

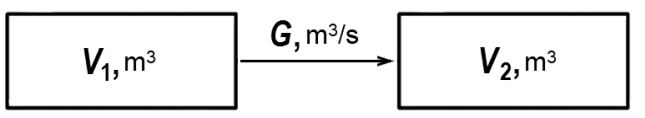

Figure 2. Scheme of the transition of GFPs between the sections of the model.

The main parameters of the model that determine the accuracy of its approximation to the reactor plant conditions are the given constants (relative rates) of the transition of nuclides from section to section. When the GFPs pass from volume $V_{1}$ to volume $V_{2}$ with flow rate $G$, the relative velocity is equal to

$$
\mu=G / V_{1}
$$

This technique makes it possible to determine the calculated activity of reference radionuclides in each block at any time after the fuel cladding failure.

At the same temperature of the LC surface at the SG inlet in each of the four loops, the absolute velocity of the GFP radionuclide release will be higher in the loop that is closer to the core sector with a leaky fuel element. This makes it possible to determine the sector where the failed fuel element is located.

The movement of the bubble that has emerged from the core is the sum of its movement with the LC flow and its displacement relative to the flow due to its rise. The diagram in Fig. 3 shows that bubbles having the ability to penetrate into the collector, will rise, during the calculated time of the LC movement from the core to the collector, only to distance equal to the collector diameter $D$. Larger bubbles rise to the LC surface in the central cavity. The bubble rise velocity was determined by the Peebles and Garber correlations (Wallis G 1972).

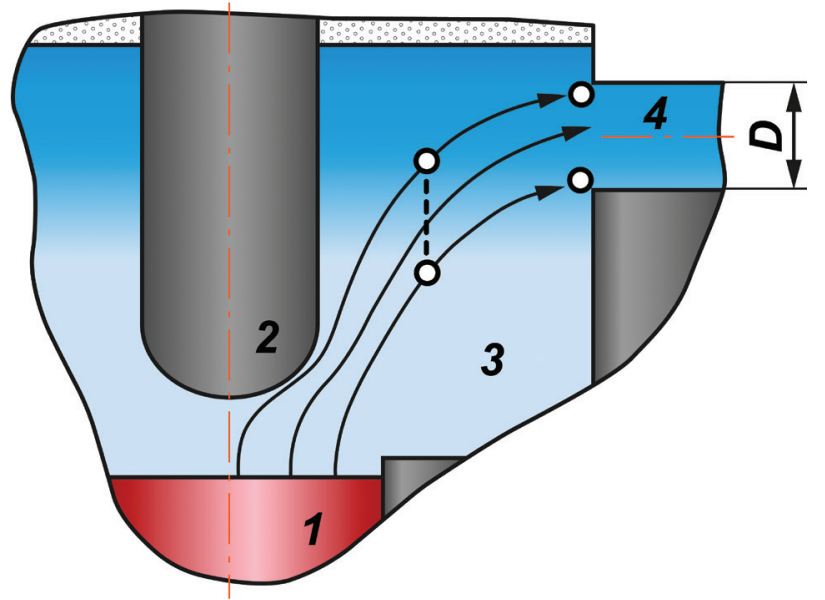

Figure 3. Scheme of bubbling up in the central cavity: 1) The core; 2) the CPS actuator column; 3) FR central cavity; 4) LC collector. 


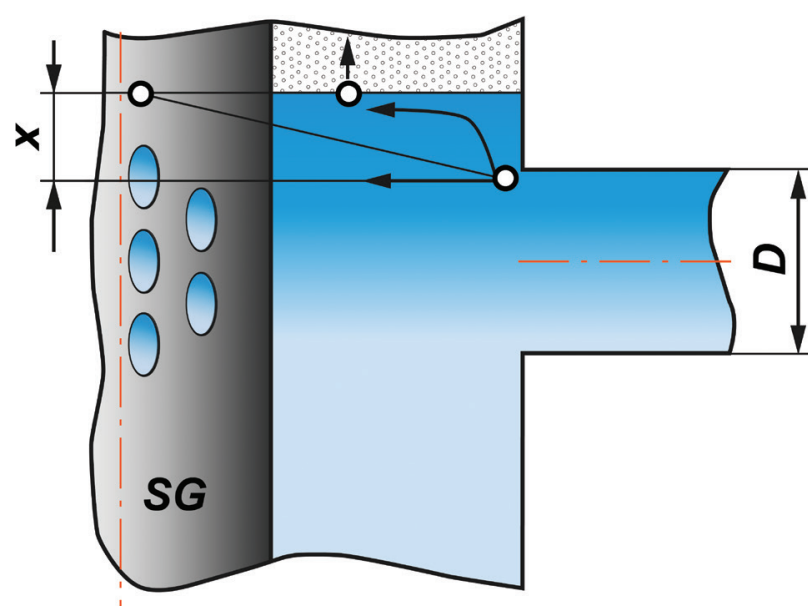

Figure 4. Scheme of bubble rise in the peripheral cavity.

Part of the bubbles in the cavity of the steam generator partition can be captured by the LC flows ascending directly to the free surface. Also, bubbles that have undergone coagulation are degassed here. In addition, the enlargement of bubbles is possible due to a significant pressure drop in the bubble when it rises from a great depth, which will result in the release of dissolved gases from the LC into the bubbles.

Part of the bubbles after leaving the collector will move with the LC horizontal flow. Figure 4 schematically shows that the bubbles, the calculated vertical displacement due to the rise of which is greater than the distance $x$ from the top of the collector to the upper opening in the $\mathrm{SG}$, are degassed before entering the SG.

The rest of the small bubbles is entrained by the LC flow into the SG, where they can be carried downward

\section{References}

- Adamov EO, Dragunov YuG, Orlov VV (2005) Mechanical Engineering of Nuclear Technology. Mashinostroenie Publ., Moscow, 960 pp. [in Russian]

- Adamov EO, Kaplienko AV, Orlov VV, Smirnov VS, Lopatkin AV (2020) Lead-cooled fast reactor BREST: from concept to technology implementation. Atomnaya Energiya [Atomic Energy] 4: 185-194. [in Russian] https://doi.org/10.1007/s10512-021-00731-w

- Babichev AP, Babushkina NA, Bratkovskiy AM (1991) Physical Quantities: Handbook. Energoatomizdat Publ., Moscow, 1232 pp. [in Russian]

- Dedul AV, Kalchenko VV, Kolik MV, Stepanov VS, Gonchar NI, Pankratov DV, Trykov LA, Efremov YuV, Yakunin SN (2009) Gamma spectrometry of shielding gas of the primary circuit of RP with HLMC as a means of operational monitoring of tightness of fuel element cladding and heat exchange surface of a steam generator. VANT. Ser. Obespechenie Bezopasnosti AES [VANT. Series: NPP Safety Ensurance] 24: 44-50. [in Russian]

- Fedotovskiy VS, Vereschagina TN, Orlov YuI (2007) Model of coagulation of bubbles introduced by a jet injector into a flow of a heavy liquid-metal coolant. Izvestia Vysshikh Uchebnykh Zawedeniy. by the LC flow, since the lead downward flow rate in the $\mathrm{SG}$ is many times higher than the bubble rise velocity. Further, such bubbles behave in the LC circuit as dissolved impurities.

After degassing, the GFPs from the gas cavities enter the cover gas take-off tubes of a special system, which makes it possible to use gamma-spectrometric analysis to determine the fact, approximate moment and approximate sector of damage to the fuel element cladding during operation at power, according to the burst of activity of the GFPs.

\section{Conclusion}

The paper describes the basic principles of monitoring the fuel cladding tightness and currently existing systems in liquid-metal-cooled reactors. It is shown that the main contribution to the background activity is made by fast neutron fission of ${ }^{238} \mathrm{U}$ contained in the coolant. The features of monitoring the fuel cladding tightness in lead-cooled reactors are presented. The GFPs can come out of leaking fuel elements both in the dissolved form and as bubbles. Consideration is given to their behavior in the coolant, the features of degassing, as well as the processes of sorption of volatile fission products and cleaning of cover gas from aerosols.

General concepts of the conditions for the transfer of GFPs in the lead coolant environment of the reactor plant under development are described, on the basis of which the methods monitoring the fuel cladding tightness will be developed by the gas activity in the gas volumes of the reactor plant.
Yadernaya Energetika [News of Higher Educational Institution. Nuclear Power Engineering] 1: 92-102. [in Russian]

- Gonchar NI (2018) Influence of uranium and thorium impurities in HLMC and structural materials of the core on the efficiency of fuel element cladding tightness control. VANT. Ser. Yaderno-Reaktornye Konstanty [VANT. Series: Nuclear Reactor Constants] 5: 176-184. [in Russian]

- Handbook on Lead-Bismuth Eutectic Alloy (2015) Handbook on Lead-Bismuth Eutectic Alloy and Lead Properties, Materials Compatibility, Thermal-hydraulics and Technologies. France. Nuclear Energy Agency Organization for Economic Co-Operation and Development Publ., 168 pp.

- Lukyanov DA, Albutova OI (2017) Method of localization of defective fuel assemblies in fast reactors. VANT. Ser. Yaderno-Reaktornye Konstanty [VANT. Series: Nuclear Reactor Constants] 3: 100-117. [in Russian]

- Lukyanov DA, Albutova OI, Zverev ID, Salyaev AV, Fadeev ID, Prokoptsov IS, Mikhaylenko MA, Guryev SA (2018) Computational and experimental substantiation of the sodium system for monitoring the tightness of the cladding of fuel elements of a promising 
commercial reactor. VANT. Ser. Yaderno-Reaktornye Konstanty [VANT. Series: Nuclear Reactor Constants] 5: 110-118. [in Russian]

- Martynov PN, Posazhennikov AM, Yagodkin IV (2007) Investigation of the behavior of aerosols in the gas circuits of reactor plants with a heavy coolant. Izvestia Vysshikh Uchebnykh Zawedeniy. Yadernaya Energetika [News of Higher Educational Institution. Nuclear Power Engineering] 1: 152-158. [in Russian]

- Mikhailov AYu, Gonchar NI (2015) Determination of the degassing characteristics of the liquid metal coolant of the primary circuit. In: Proc. of the Sci.-Techn. Conf. "Thermal Physics of Fast Nuclear Reactors (Thermophysics-2014)”. GNTs RF-FEI Publ., Obninsk, 437-444. [in Russian]

- Naumov VS, Konovalov EE (2018) Sorption of radionuclides formed in the primary circuits of reactors with $\mathrm{Pb}-\mathrm{Bi}$ coolant, the surface of structural materials of the core. Radiokhimiya [Radiochemistry] 2: 167-174. [in Russian] https://doi.org/10.1134/ S1066362218020091

- NP-082-07 (2008) Federalnaya Sluzhba po Ekologicheskomu, Tekhnologicheskomu i Atomnomu Nadzoru. Nuclear Safety Rules for Reactor Facilities of Nuclear Power Plants. Yadernaya i Radiatsionnaya Bezopasnost [Nuclear and Radiation Safety] 1: 52-77. [in Russian]

- Posazhennikov AM, Yagodkin IV, Papovyants AK, Grishin AG, Isayev AYu (2015) Cleaning of protective gas from aerosols of heavy liquid metal coolants. VANT. Ser. Yaderno-Reaktornye Konstanty [VANT. Series: Nuclear Reactor Constants] 2: 102-119.
- Putilov KA (1963) Physics Course. Volume I. Mechanics, Acoustics, Molecular Physics, Thermodynamics. Fizmatgiz Publ., Moscow, 560 pp. [in Russian]

- SanPiN 2.6.1.24-03 (2021) Sanitary rules for the design and operation of nuclear power plants (SP AS-03). https://docs.cntd.ru/document/901862274 [accessed Mar. 17, 2021] [in Russian]

- Skovorodko SN, Mozgovoy AG (2010) Solubility of inert gases in heavy liquid metal coolants at high temperatures. Teplofizika Vysokikh Temperatur [Thermal Physics of High Temperatures] 4: 633-637. [in Russian] https://doi.org/10.1134/S0018151X10040206

- Solubility data series (1979) Volume 2. Krypton, Xenon and RadonGas Solubilities. Pergamon press, Great Britain: XVIII.

- Vasyukhno VP, Dubenkov NE, Lemekhov VV, Morkin MS, Khacheresov GA, Rychkov VS, Shushlebin VV (2017) Investigation of the processes of mass transfer of fuel fission products and activation products of lead coolant impurities at a complex of leadgas loop installations. In: Proc. of the Conf. "Nuclear innovations". NIKIET JSC Publ., Moscow, 322-330. [in Russian]

- Vereschagina TN, Lemehov VV, Morkin MS (2020) Lead-cooled gas lift probe hydraulics. In: Proc. of the Sci.-Techn. Conf. "Thermophysics-2020”. GNTs RF-FEI Publ., Obninsk, 136. [in Russian]

- Veselkin AP, Yegorov YuA (1976) Engineering Calculation of the Protection of Nuclear Power Plants. Atomizdat Publ., Moscow, 296 pp. [in Russian]

- Wallis G (1972) One-Dimensional Two-Phase Flows. Mir Publ., Moscow, 440 pp. [in Russian] 